\title{
The roles of mechanosensitive ion channels and associated downstream MAPK signaling pathways in PDLC mechanotransduction
}

\author{
YUN SHEN, YONGCHU PAN, SHUYU GUO, LIAN SUN, CHI ZHANG and LIN WANG \\ Institute of Stomatology, Jiangsu Key Laboratory of Oral Diseases, Nanjing Medical University, \\ Nanjing, Jiangsu 210029, P.R. China
}

Received March 26, 2019; Accepted February 5, 2020

DOI: $10.3892 / \mathrm{mmr} .2020 .11006$

\begin{abstract}
The present study aimed to investigate whether the cytoskeleton, the Piezol ion channel and the transient receptor potential cation channel subfamily $\mathrm{V}$ member 4 (TRPV4) ion channel are equally functional in the mechanotransduction of periodontal ligament cells (PDLCs) and to reveal the interplay of these mechanically sensitive ion channels (MSCs). Human PDLCs (hPDLCs) were pretreated with cytochalasin D (the inhibitor of actin polymerization), GsMTx4 (the antagonist of Piezo1) and GSK205 (the antagonist of TRPV4), and then subjected to periodic mechanical loading. The expression levels of macrophage colony stimulating factor (M-CSF), receptor activator of NF- $\kappa$ B ligand (RANKL) and cyclooxygenase-2 (COX2) in hPDLCs were detected via western blotting. Osteoblast mineralization induction capacity of the hPDLCs was also studied and the mitogen-activated protein kinase (MAPK) expression profile was determined via protein microarray. The expression of Piezo1 and TRPV4 in the PDLCs was significantly increased at $8 \mathrm{~h}$ after loading. These differences in expression were accompanied by increased expression of M-CSF, RANKL and COX2. Compared with the control group, key PDLC biomarkers were suppressed after mechanical loading following treatment with the inhibitors of Piezo1 (GsMTx4) and TRPV4 (GSK205). The phosphorylated-MAPK protein array showed differential biomarker profiles among all groups. The present study suggested that both MSCs and the cytoskeleton participated as mechanical sensors, and did so independently in hPDLC mechanotransduction. Furthermore, the Piezol ion channel may transmit mechanical signals via the ERK signaling pathway; however, the TRPV4 channel may function via alternative signaling pathways.
\end{abstract}

Correspondence to: Dr Lin Wang, Institute of Stomatology, Jiangsu Key Laboratory of Oral Diseases, Nanjing Medical University, 136 Hanzhong Road, Nanjing, Jiangsu 210029, P.R. China E-mail:1w603@njmu.edu.cn

Key words: periodontal ligament cells, Piezol, transient receptor potential cation channel subfamily $\mathrm{V}$ member 4, mitogen-activated protein kinase pathway, mechanotransduction

\section{Introduction}

Dental implants and ankylosed teeth cannot be moved by orthodontic force, which highlights the importance of periodontal ligament cells (PDLCs) in orthodontic tooth movement (1).

PDLCs are mechanically sensitive cells that may be induced by mechanical force, and they express a series of cytokines, including macrophage colony stimulating factor (M-CSF), receptor activator of $N F-\kappa B$ ligand (RANKL), osteoprotegerin (OPG) and inflammatory factors $(2,3)$. The combined functional interventions that result from these factors serve to control the development of alveolar bone $(4,5)$. However, the processes underlying the transduction of the associated mechanical signals to elicited biological signals, which is mediated by PDLCs, remain to be elucidated.

To date, 2 mechanotransduction mechanisms in the cells have captured considerable attention. First, attention has focused on the cytoskeletal-integrin-focal adhesion pathway, which receives and delivers the signals following transformation of microfilaments and microtubules $(6,7)$. Second, attention has also focused on the mechanically sensitive ion channels (MSCs) on the surface of the cytomembrane, which transduce signals by increasing membrane permeability of the ion channels and triggering the influx of extracellular calcium (8). It has been proposed that the activation of calcium ion channels and subsequent calcium influx is associated with cytoskeletal transformation on induction by stress stimuli (9). However, this process was contradicted by Cox et al (10), which states that the integrity of the cytoskeleton is irrelevant in the context of Piezol ion channel function. The functional roles played by MSCs in orthodontic force-induced PDLC activation and the relationship between these two types of mechanotransduction have been poorly studied.

Piezo 1 and transient receptor potential cation channel subfamily V member 4 (TRPV4) are two typical MSCs that have received widespread attention from the research community. Piezol was first identified in a mouse neuroblastoma cell line; it was determined to respond to mechanical stimuli in as little as $5 \mathrm{msec}$ and trigger calcium influx into the cells (11). A distinctive feature of the microscopic structure of Piezo1 is the flexible blades region, which is proposed to rotate and expose the central ion-conducting pore under mechanical stimulus (12). 
Distinct from Piezo1, TRPV4 was initially recognized as an osmotically activated channel (13). Further studies identified that TRPV4 could be activated by fluid shear stress and phorbol ester $(14,15)$. However, the gating mechanisms of TRPV4 remain to be elucidated. Although Piezol and TRPV4 are found in several mechanically sensitive cells (16-18), the downstream signal transduction pathways remain unknown.

Mitogen-activated protein kinase (MAPK) refers to a group of protein kinases that are associated with Piezol and the TRPV4 channel $(19,20)$. It has been identified that an ERK1/2 inhibitor decreased the expression of Piezol in neonatal rat ventricular myocytes, whereas this effect was not observed when p38 and JNK inhibitors were applied (21). Additionally, the p38 inhibitor SB203580 enhanced the expression of TRPV4 in the dorsal root ganglion (22). Collectively, these observations suggest that MAPKs may participate in signal transduction pathways downstream of MSCs under conditions of mechanical loading.

In the present study, human primary PDLCs were subjected to stretch using a Flexcell device, leading to a model of stress-induced transformation. The roles played by MSCs in PDLC mechanotransduction were functionally analyzed by deconstructing the cytoskeleton using cytochalasin $\mathrm{D}$ (cytoD), or by blocking the Piezol channel using GsMTx4 or the TRPV4 channel using GSK205. The expression profiles of the MAPK signaling pathway in PDLCs when both of the MSCs were specifically blocked by targeted inhibition was also investigated.

\section{Materials and methods}

Cell culture. Human PDLCs were obtained from premolars that were extracted from 4 young donors for orthodontic consultation and treatment at the Jiangsu Stomatological Hospital. All donors were healthy ethnic Han Chinese females between 12 and 14 years old. The donors and their legal guardians were fully informed of the purpose of this study and provided written informed consent. All human experimental protocols were approved by the Ethics Committee of Shanghai Tenth People's Hospital [policy no. 2008 (20)]. The periodontal ligament was scraped from the root surfaces of the teeth and digested with collagenase type I (Sigma-Aldrich; Merck KGaA) for $30 \mathrm{~min}$ at $37^{\circ} \mathrm{C}$. Cells were collected and resuspended in low-glucose DMEM (HyClone; GE Healthcare Life Sciences) that was supplemented with $15 \%$ FBS (ScienCell Research Laboratories, Inc.), $100 \mathrm{U} / \mathrm{ml}$ penicillin-G and $100 \mu \mathrm{g} / \mathrm{ml}$ streptomycin sulfate (HyClone; GE Healthcare Life Sciences). Cells were passaged when they reached $\sim 90 \%$ confluence, and those from passages 3-5 were used in subsequent experiments.

Primary mouse osteoblasts were isolated from 20 2-3-day-old BALB/c neonatal female mice (Beijing Vital River Laboratory Animal Technology); animals were sacrificed on arrival. All animal experimental protocols were approved by the Ethics Committee of Shanghai Tenth People's Hospital (policy no. SHDSYY-2017-2473). The calvarial bones of the mice were cut into fractions and digested using $0.25 \%$ trypsin for $30 \mathrm{~min}$ and $1 \mathrm{mg} / \mathrm{ml}$ collagenase type II for $10 \mathrm{~min}$ (Sigma-Aldrich; Merck KGaA) at $37^{\circ} \mathrm{C}$. Following digestion, the fractions were resuspended in DMEM supplemented with $10 \% \mathrm{FBS}$ and incubated at $37^{\circ} \mathrm{C}$ in a humidified atmosphere of
$95 \%$ air and $5 \% \mathrm{CO}_{2}$. Cells were passaged when they reached 90\% confluence, and those from passages 3-5 were used in subsequent experiments.

Cell loading. Human periodontal ligament cells (hPDLCs) were plated in BioFlex culture plates (type I collagen-coated; Flexcell International Corporation) and divided into four groups. The first was a control group, in which cells were loaded onto a Flexcell tension system (FX-5000T, Flexcell International Corporation) without any treatment. The second were cells in the cytoD group, which were pretreated with cytoD (Sigma-Aldrich; Merck KGaA) at a concentration of $5 \mu \mathrm{g} / \mathrm{ml}$ to depolymerize F-actin in the cells and incubated at $37^{\circ} \mathrm{C}$ for $20 \mathrm{~min}$, cells were refreshed by addition of fresh culture medium without cytoD. For the third (GSK205) and fourth (GsMTx4) groups, fresh medium respectively containing $30 \mu \mathrm{M}$ GSK205 (cat. no. 616522; Merck KGaA) and 500 nM GsMTx4 (cat. no. ab141871; Abcam) were added separately before cell loading to suppress the TRPV4 and Piezol channels, and cells were subjected to these compounds during loading Next, the plates were set on the FX-5000T. Then, $15 \%$ stretch was applied onto the cells for 4,8 and $12 \mathrm{~h}$. According to the study of Lu et al (9) on three different cell types, a stretch time duration of $<3 \mathrm{sec}$ fails to initiate calcium influx. Consequently, a $0.25-\mathrm{Hz}$ square waveform of periodic loading, which persisted for $3 \mathrm{sec}$ with a 1-sec release, was selected for the present study.

Calcium measurements. hPDLCs in all groups were washed three times in PBS following the stretch procedure. Then, the cells were treated with $1 \mu \mathrm{M}$ Fluo-4AM (Beyotime Institute of Biotechnology) and incubated for $20 \mathrm{~min}$ in the dark at $37^{\circ} \mathrm{C}$. Afterwards, the cells were washed three times in PBS and observed by fluorescence microscopy (DMI3000B; Leica Microsystems $\mathrm{GmbH}$ ). The fluorescence intensity was measured using ImageJ 2X 2.1.4.6 software (National Institutes of Health). To do the measurements, the image was first converted to 8-bit grayscale and then inverted. The threshold was then set to ensure that only the cell regions were selected, and measurements were performed to obtain the area of the cells, the integrated intensity of the region and the average gray value of the selected region. The average gray value of each image was used for statistical analysis.

Cytoskeletal fluorescence staining. hPDLCs were washed three times in PBS following stretching and fixed in $4 \%$ paraformaldehyde (Beijing Leagene Biotech Co Ltd.) for $15 \mathrm{~min}$ at room temperature. Thereafter, the cells were permeabilized in $0.5 \%$ Triton $\mathrm{X}-100$ for $15 \mathrm{~min}$ at room temperature and then rinsed three times in PBS. ActinGreen ${ }^{\text {TM }} 488$ ReadyProbes $^{\circledR}$ Reagent (Thermo Fisher Scientific, Inc.) was added to all plates, after which they were incubated at room temperature for $30 \mathrm{~min}$ in the dark. Finally, cells were washed three times in PBS and observed by fluorescence microscopy at x200 magnification (DMI3000B; Leica Microsystems GmbH). The average gray value of the cells were also measured by the method described above.

Reverse transcription-quantitative $(R T-q) P C R$. After $0,4,8$ or $12 \mathrm{~h}$ loading, total RNA was extracted from the PDLCs using 
Table I. Sequences of primers used in the present study.

\begin{tabular}{ll} 
mRNA & \multicolumn{1}{c}{ Primer sequence } \\
\hline RANKL & Forward: 5'-ACCGACATCCCATCTGGTT-3' \\
OPG & Reverse: 5'-GCCATCCTGATTAACTATTAGTT-3' \\
& Forward: 5'-AAGCCTTCTCTAACCTCTCC-3' \\
COX2 & Reverse: 5'-GCCCTCGCTTATGATCTGTC-3' \\
& Forward: 5'-TTGAAATGGCAGTTGATTCCTTT-3' \\
Piezo1 & Reverse: 5'-TATCCTCTTTCTCAGGGTGCTTG-3' \\
& Forward: 5'-GGCAACATGAGGGAGTTCATTAACTC-3' \\
TRPV4 & Reverse: 5'-TTCTCCGTCAGGTAGTTGACAATGTG-3' \\
GAPDH & Forward: 5'-GGCAACTTCCTCACCAAGA-3' \\
& Reverse: 5'-GGGTATTTCTTCTCTGTCTCT-3' \\
& Forward: 5'-GGCACAGTCAAGGCTGAGAATG-3' \\
& Reverse: 5'-ATGGTGGTGAAGACGCCAGTA-3'
\end{tabular}

COX2, cyclooxygenase-2; RANKL, receptor activator of NF- $\mathrm{B}$ ligand; OPG, osteoprotegerin; TRPV4, transient receptor potential cation channel subfamily $\mathrm{V}$ member 4.

the Tiangen RNAprep pure kit (Tiangen Biotech Co., Ltd.). cDNA was obtained with the PrimeScript ${ }^{\mathrm{TM}}$ RT Master Mix reagent (Takara Bio, Inc.) following the manufacturer's instructions. The cDNA was added to a $20-\mu 1$ system for qPCR using the SYBR Green Reaction kit (Roche Diagnostics) using the ABI Prism 7300 Real-Time PCR system (Applied Biosystems; Thermo Fisher Scientific, Inc.). The reaction was carried out under the following conditions: $94^{\circ} \mathrm{C}$ for $2 \mathrm{~min}$, then 40 cycles of $94^{\circ} \mathrm{C}$ for $30 \mathrm{sec}$ and $55^{\circ} \mathrm{C}$ for $1 \mathrm{~min}$. The comparative $2^{-\Delta \Delta \mathrm{Cq}}$ method was used to calculate the fold-change of mRNA (23). Primers used are listed in Table I.

Osteoblast mineralization induction. Murine osteoblasts that were derived from passages 3-5 were seeded into 24-well plates. Culture media from all four PDLC treatment groups, which were obtained following loading, were added to the plates when the cells reached $\sim 90 \%$ confluence. The media was changed every three days. After 10 days of culture, cells were fixed in $95 \%$ ethanol at room temperature for $15 \mathrm{~min}$, and then stained with $0.2 \%$ Alizarin red at room temperature for $5 \mathrm{~min}$.

Western blot analysis. Cells obtained from the control group, the cytoD group and the GSK205 and GsMTx4 groups were washed in PBS following loading, following which they were lysed and their total protein was extracted using a whole-cell lysis kit (Wanleibio Co., Ltd.). Protein concentration was determined with an enhanced bicinchoninic acid protein assay reagent kit (cat. no. P0009; Beyotime Institute of Biotechnology). Proteins were subjected to electrophoresis in a $12 \%$ SDS polyacrylamide gel (40 $\mu \mathrm{g}$ protein per lane) and then transferred on to a PVDF immunoblotting membrane (EMD Millipore). Then, $5 \mu 1$ molecular weight standards (cat. no. 26616; Fermentas; Thermo Fisher Scientific, Inc.) was loaded onto the gels as a reference. The membrane was blocked in $5 \%$ skimmed milk for $2 \mathrm{~h}$ at room temperature and incubated with cyclooxygenase-2 (COX2; 1:500; cat. no. ab179800;
Abcam), RANKL (1:500; cat. no. ab65024; Abcam) and OPG (1:500; cat. no. ab183910; Abcam) primary antibodies at $4^{\circ} \mathrm{C}$ overnight. The membrane was washed six times in Tris-buffered saline containing $0.05 \%$ Tween 20 (TBST) for $5 \mathrm{~min}$ per wash. The membrane was then incubated in secondary antibodies (1:1,000; cat. no. WLA023; Wanleibio, Co., Ltd.) for $45 \mathrm{~min}$ at $37^{\circ} \mathrm{C}$ and washed six times in TBST. The membrane-immobilized protein bands were detected with chemiluminescent detection reagents (cat. no. WBKLS0100; EMD Millipore) in the WD-9413 fluorescence imaging system (Beijing Liuyi Biotechnology Co., Ltd.). The gray values for the visible bands were analyzed with the Gel-Pro Analyzer software 4.0 (Meyer Instruments, Inc.).

Expression profiling using protein array. Whole-cell proteins extracted from PDLCs in all four groups after the loading procedure were screened for their protein expression profiles using a Human Phospho-MAPK array kit (cat. no. ARY002B; R\&D Systems, Inc.) according to the manufacturer's instructions, variations of $>20 \%$ are considered potential candidates in the mechanotransduction of PDLCs.

Statistical analysis. Each experiment was repeated three times independently and data are presented as the mean \pm SD. Statistical analyses were performed by one-way analysis of variance using GraphPad Prism 6 software (GraphPad Software, Inc.). Tukey's analysis was performed for multiple comparison tests. $\mathrm{P}<0.05$ was considered to indicate a statistically significant difference.

\section{Results}

Increased $m R N A$ expression of COX2, RANKL, OPG, Piezol and TRPV4 in PDLCs following periodic mechanical loading. The mRNA levels of key biomarkers, including COX2, RANKL and OPG, were increased after periodic loading at $0.25 \mathrm{~Hz}$, indicating that the PDLCs were activated after stretch 
(Fig. 1). Additionally, the increased expression of TRPV4 and Piezol suggested that both ion channels were related to PDLC activation following loading (Fig. 1). However, it was noted that while most of the expression levels peaked at $8 \mathrm{~h}$, additional stretch loading reduced PDLC activation, which could be attributed to the plate membrane losing its elasticity. Another reason for this observation may have been due to adaptation of the mechanosensitive cells and attenuation of the mechanotransduction process following loading, as was discussed in our previous study (24).

GsMTx4 and GSK205 do not interfere with cytoskeletal reconstruction in PDLCs, while CytoD does not affect calcium influx following loading. Fluorescent images showed that the intracellular calcium concentrations of PDLCs treated with GsMTx4 and GSK205 decreased compared with the control group following loading; however, cells that were treated with cytoD exhibited a fluorescence intensity similar to that of the control group (Fig. 2). This result suggested that the roles played by MSCs were independent of cytoskeletal integrity. The fluorescent images of the cytoskeleton showed that in cells that were pretreated with cytoD, a wrinkled appearance was noted and the intensity of the fluorescence decreased. By contrast, neither GsMTx4- nor GSK205-treated cells exhibited any differences in fluorescence intensity or cell morphology (Fig. 3).

Destruction of the cytoskeleton and inhibition of mechanosensitive ion channels may compromise PDLC activation induced by periodic stretching. The biomarkers COX2, RANKL and OPG were all secreted by PDLCs after mechanical loading. COX2 is the rate-limiting enzyme that converts arachidonic acid to prostaglandins (PGs), which can regulate local inflammation and play a key role in the reconstruction of alveolar bone under orthodontic forces (25). The ratio of RANKL/OPG directly controls the balance between osteogenesis and osteoclastogenesis (26). It has been established that increased expression of these factors in PDLCs is induced by mechanical loading $(27,28)$.

In the present study, the observed increases in biomarker expression were compromised by cytoD, GsMTx 4 and GSK205 treatment (Fig. 4), which indicated that the efficiency of mechanotransduction was reduced. The results suggested that the cytoskeleton, Piezo1 and TRPV4 may affect signal transduction in PDLCs after stretch loading. The culture of osteoblasts with loaded PDLC supernatants also demonstrated that the supernatant of the cells treated with the selected inhibitors exhibited a diminished capacity to promote osteogenesis (Fig. 5).

Roles played by cytoskeletal and mechanosensitive ion channels in PDLC mechanotransduction may be mediated by MAPK pathways. Although all three inhibitors decreased PDLC activation that was otherwise induced by stretch, the profiles of MAPK-associated proteins in each group were observed to differ. Following a microarray analysis of phosphorylated MAPKs, the three groups of cells that were treated by the inhibitors were compared with the control group. Fold changes in the phosphorylation of proteins in each group are shown in Fig. 6.

\section{Discussion}

It has been established that PDLCs are mechanosensitive cells that secrete multiple cytokines to regulate the reconstruction of local alveolar bone $(28,29)$. This mechanism could be considered the foundation of their role in orthodontic tooth movement $(29,30)$. PDLCs at the pressure side are capable of continuous RANKL expression (28). Additionally, increased expression of PGE2, tumor necrosis factor- $\alpha$, interleukin (IL)-1 $\beta$, IL-2, IL-6, IL-8 and M-CSF has been detected in periodontal ligament loaded by orthodontic force $(31,32)$. Tension loading can also activate PDLCs (32).

In the present study, the expression of COX2, RANKL and OPG in PDLCs was elevated following tension loading, which corresponds with results obtained from previously published studies (27). ELISA was not performed in the present study, as the aim of employing RT-qPCR was to find the time point of peak molecular expression of the genes of interest. In addition, changes in mRNA levels were considered to be more sensitive than the estimation of protein levels by assays such as ELISA.

Among the biomarkers, $\mathrm{COX} 2$ regulates local inflammation by regulating the synthesis of PGs (25). Increased COX2 expression has been detected in multiple mechanosensitive cells, and is considered to be an indication of extraneous stimulation intensity (24). RANKL and OPG are factors that play opposing roles and both participate in bone metabolism $(26,33)$. Increases in these factors suggested that PDLCs were activated by periodic stretch loading. Additionally, increased TRPV4 and Piezol expression implied that calcium ion channels may be involved in mechanotransduction of stretch loading. When PDLCs are subjected to stress, increased levels of TRPV4 and Piezo1 enable greater efficiency of the cells in the mechanotransduction process and regulate their biological adaptation to the environment (34). Similar feedback regulation can be detected in cytoskeletal reconstruction following loading, as reported in our previous study (24). The strengthening of mechanical sensors in the cells following loading may be attributable to positive feedback mechanisms, which requires further investigation.

The roles played by the cytoskeleton in the process of stress-strain-cell signal transduction have been established (35-37). MSCs, particularly TRPV4 and Piezol, have also been considered key factors in mechanotransduction $(8,38)$. It has been suggested that mechanical loading can induce calcium influx and calcium-dependent cytoskeletal reorganization (39). However, it remains to be determined as to whether cytoskeletal transformation induced by stress can activate the calcium ion channels on the cytomembrane, or whether the calcium influx that is initiated by calcium ion channels can regulate cytoskeletal reorganization through downstream signaling pathways.

A previous study employing the patch-clamp assay revealed that the integrity of the cytoskeleton is not essential for the functioning of the Piezol channel (10). Conversely, certain investigators do not trust the patch-clamp database, as the surfaces of the cytomembrane are subjected to continuous tension forces during an experimental study, which may lead to results that differ from those found in normal cells (34). By contrast, another study found decreased calcium influx when induced pluripotent stem cells were pretreated with cytoD 

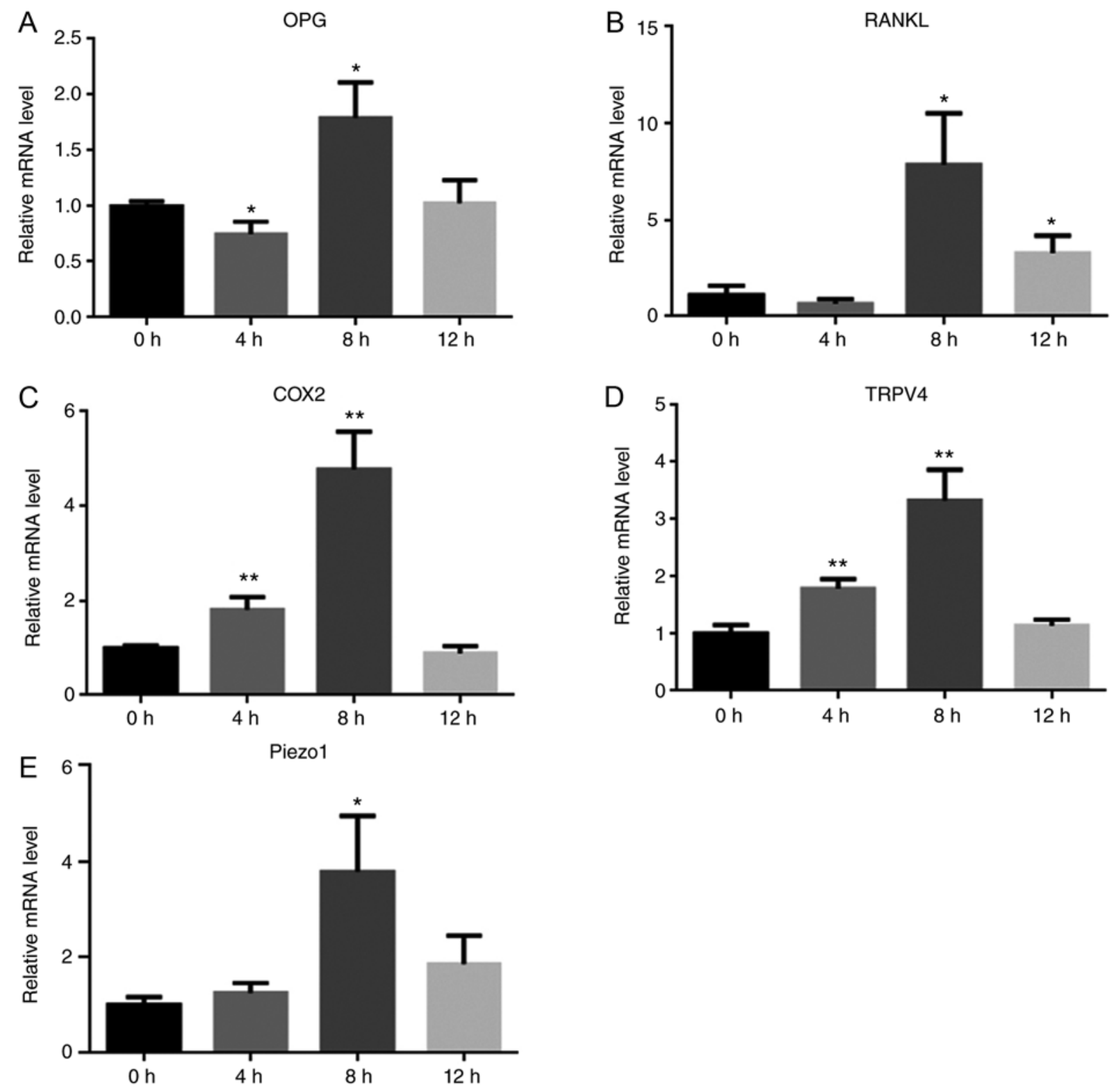

Figure 1. Stretch loading induces the mRNA expression of COX2, RANKL, OPG, TRPV4 and Piezo1 in PDLCs. mRNA expression patterns of COX2, RANKL, OPG, TRPV4 and Piezo1 in PDLCs following loading. (A) OPG, (B) RANKL, (C) COX2, (D) TRPV4 and (E) for Piezol. "P<0.05, ${ }^{* *} \mathrm{P}<0.01 \mathrm{vs.} 0 \mathrm{~h}$. PDLC, periodontal ligament cell; COX2, cyclooxygenase-2; RANKL, receptor activator of NF- $\mathrm{BB}$ ligand; OPG, osteoprotegerin; TRPV4, transient receptor potential cation channel subfamily $\mathrm{V}$ member 4.

prior to application of $20 \%$ stretch force; such effects were not observed when $10-15 \%$ stretch was used (9).

In the present study, $15 \%$ stretch was loaded onto the PDLCs, and no effect on calcium influx was found by treatment with cytoD, consistent with the abovementioned studies. Additionally, such contradictions as are described in previous studies may imply that the Piezol channel, unlike TRPV4, is not involved in cytoskeletal reconstruction. In support of this, Matthews et al (40) reported that the force applied to $\beta-1$ integrins could activate the TRPV4 ion channel. In summary, no meaningful interactions between the cytoskeleton and these two ion channels were detected by the present study.

Fluxes in calcium ion concentrations play a critical role in cell-mediated biological activities that are regulated by calcium ion channels at the cytoplasmic membrane $(41,42)$, thus enabling MSCs to regulate the cell cycle, proliferation, differentiation and apoptosis through calcium-dependent pathways upon application of a mechanical force (43). In the present study, administration of Piezol and TRPV4 antagonists decreased intracellular calcium concentrations following loading. The antagonists also attenuated the activation of three important biomarkers, RANKL, OPG and COX2, in PDLCs. The results suggested that both Piezo1 and TRPV4 are key factors in PDLC mechanotransduction. Jin et al (8) reported the effect of Piezol in this process; however, the mechanical loading in that study was performed under conditions of static pressure. This, combined with the results of the present study, suggests that both tension and pressure forces are recognized by the Piezol channel, which could initiate the expression of key cellular signals. By contrast, TRPV4 is a special ion channel that possesses similar permeability for the divalent cations $\mathrm{Ca}^{2+}, \mathrm{Sr}^{2+}, \mathrm{Mg}^{2+}$ and $\mathrm{Ba}^{2+}$; however, under physiologic 

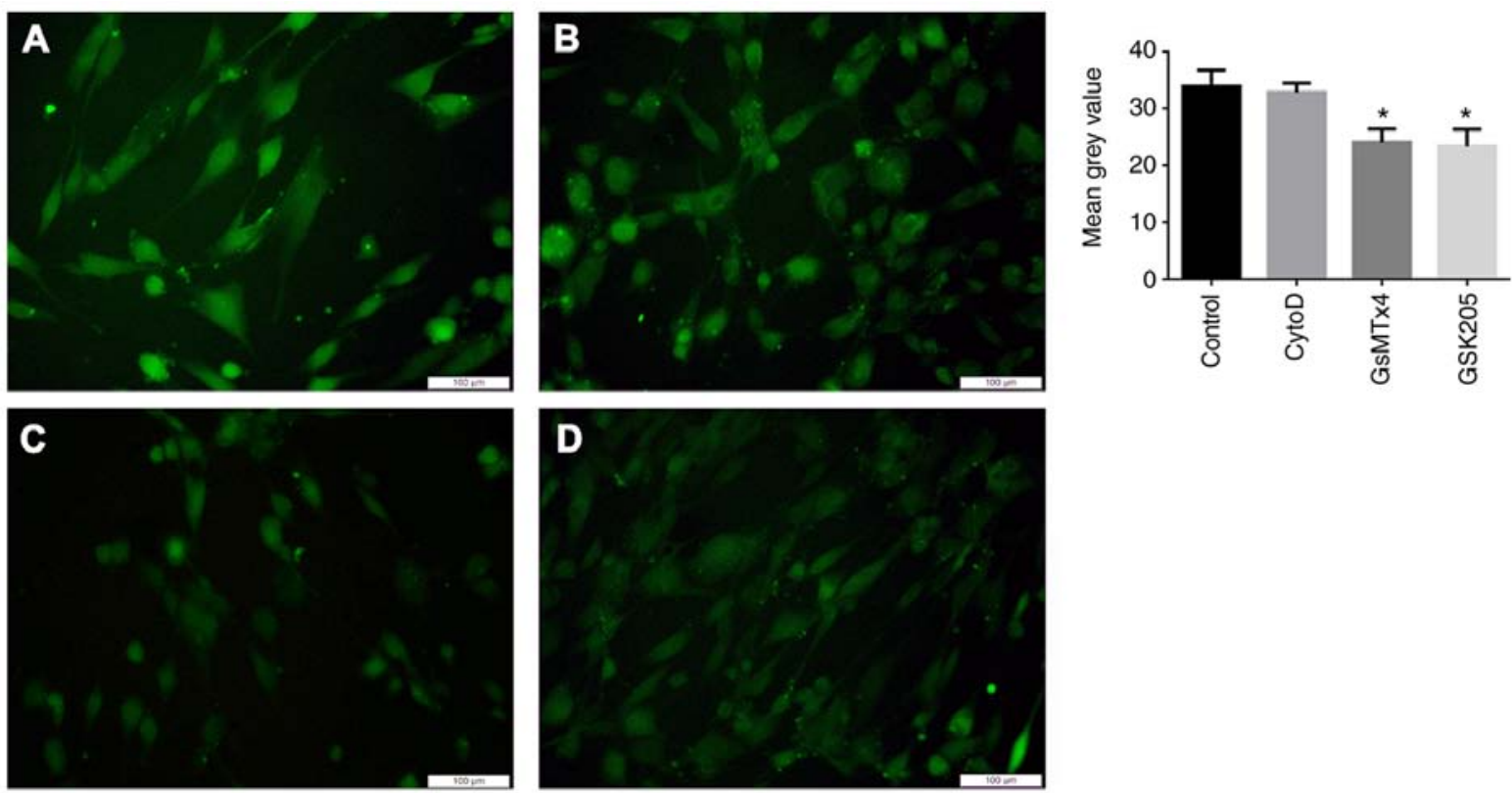

Figure 2. Calcium influx is inhibited by GsMTx4 and GSK205 after $8 \mathrm{~h}$ of loading. (A) Control group, (B) cytoD-pretreated group, (C) GsMTx4-treated group and (D) GSK205-treated group. The histogram shows quantified fluorescence intensity in each group. Compared with the group loaded normally (as the control), equivalent fluorescence intensity was emitted by cells that were pretreated with cytoD and comparatively less fluorescence was observed in the GsMTx4- and GSK205-treated groups. ${ }^{*} \mathrm{P}<0.05$ vs. Control. cytoD, cytochalasin D.
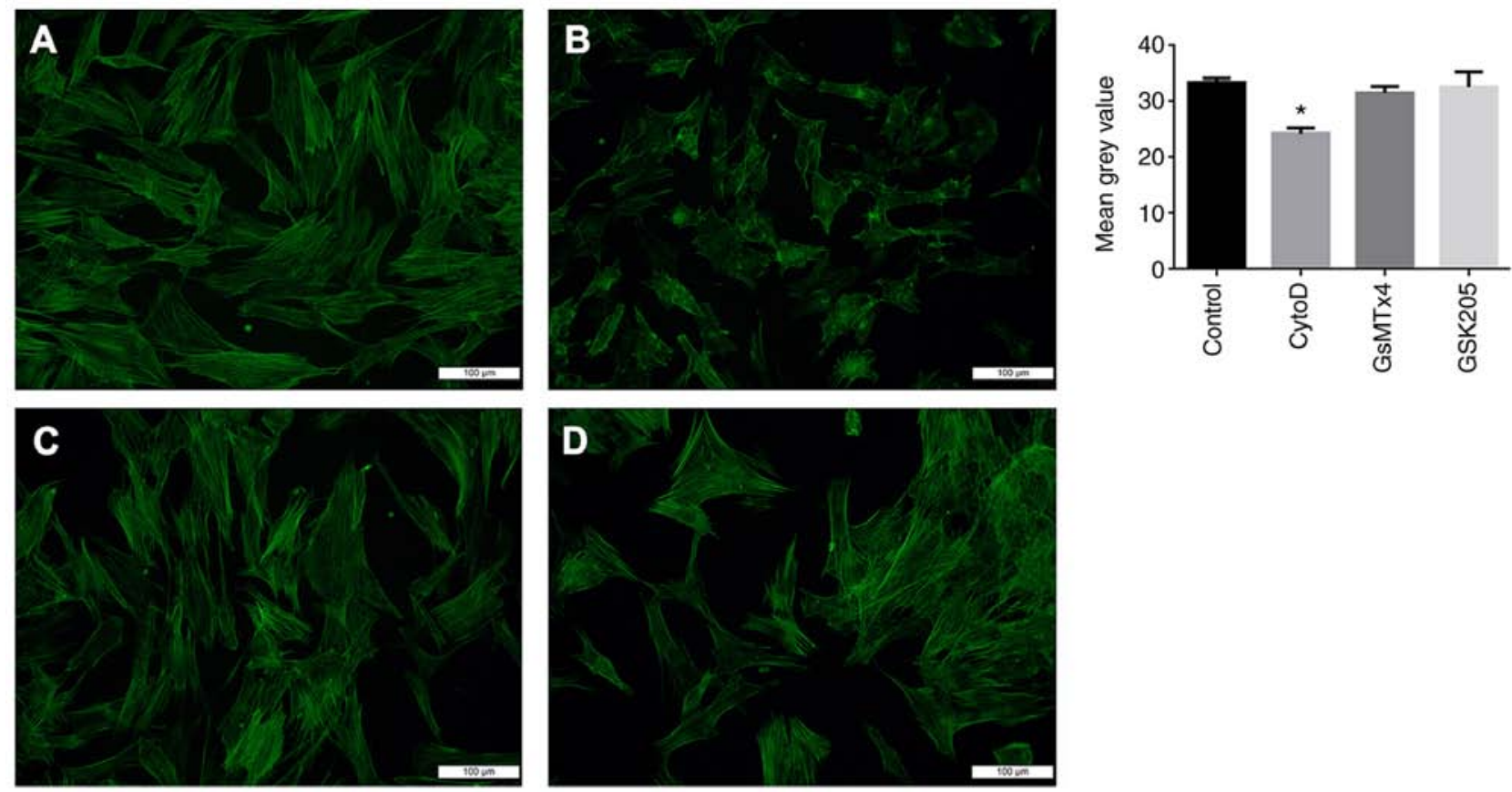

Figure 3. Structural integrity of the cytoskeleton is compromised by cytoD after $8 \mathrm{~h}$ of loading. (A) Control group, (B) cytoD-pretreated group, (C) GsMTx4-treated group and (D) GSK205-treated group. The histogram shows quantified fluorescence intensity in each group. CytoD destroyed F-actin expression in PDLCs; it was found that the fluorescence was reduced and the fibers were irregularly arranged. In the GsMTx4- and GSK205-treated groups, no significant difference was observed. ${ }^{*}<0.05$ vs. Control. cytoD, cytochalasin D.

conditions, $\mathrm{Ca}^{2+}$ is the predominant ion that migrates through the TRPV4 channel $(13,44)$.

Son et al (38) studied the role of TRPV4 in PDLCs and found that when TRPV4 was activated by osmotic pressure, calcium influx was otherwise evoked. Meanwhile, although the expression of RANKL was increased, it was found that OPG expression remained unchanged (38). In the present study, the expression levels of both RANKL and OPG were decreased. This could be formally attributed to the fact that the mechanical force applied in the present study was a periodic stretch force. These conditions differed from those reported in the previous study (38) and could have resulted in diverse effects on TRPV4. Analysis of existing data has revealed that, although both Piezol and TRPV4 participate in mechanotransduction, they differ in 

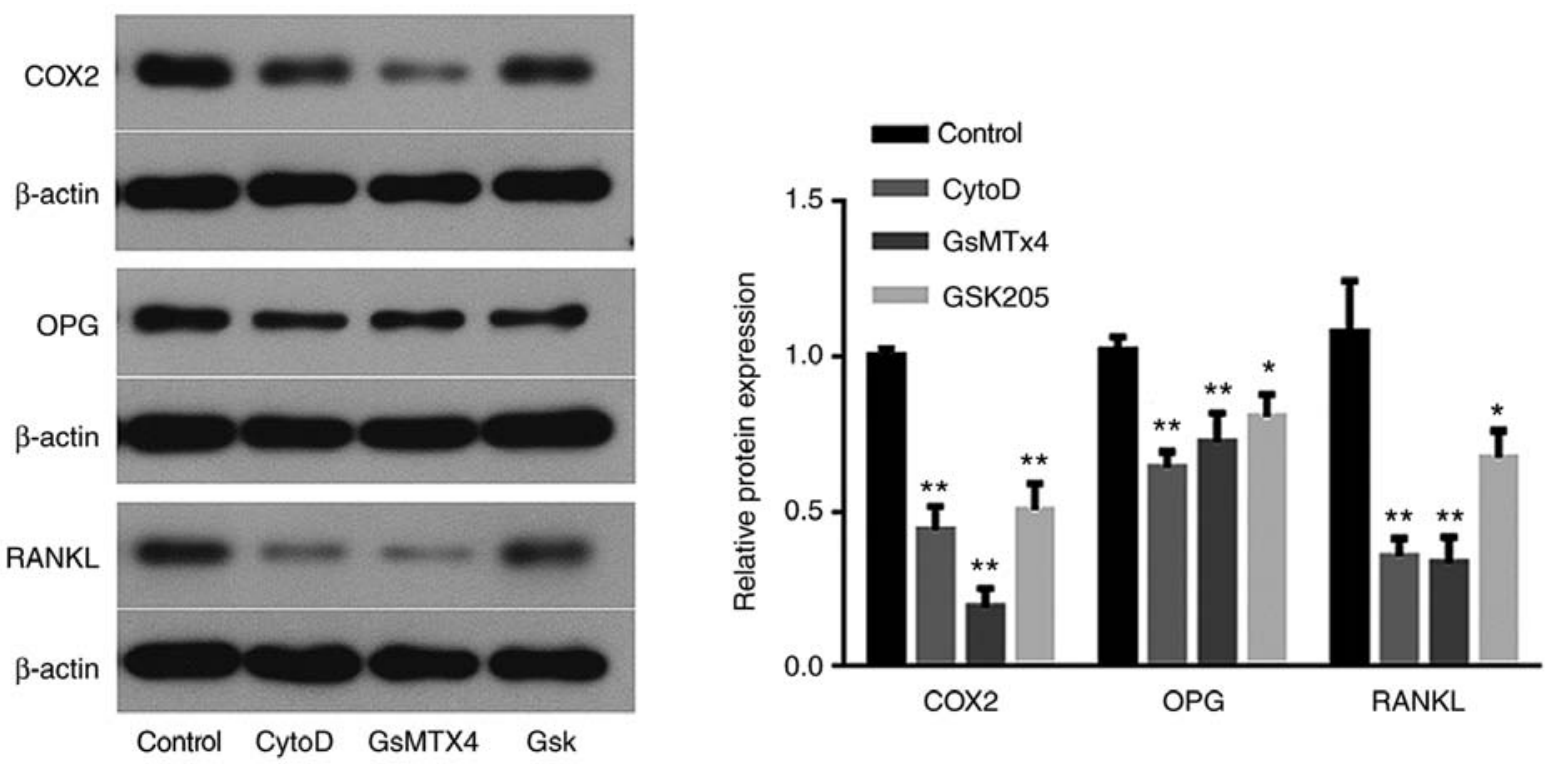

Figure 4. Expression levels of COX2, RANKL and OPG are downregulated by the inhibitors after $8 \mathrm{~h}$ of loading. Following treatment of cells with the inhibitors, the expression of COX2, RANKL and OPG was decreased compared with that found in the normally loaded group. " $\mathrm{P}<0.05$, " $\mathrm{P}<0.01$ vs. Control. COX2, cyclooxygenase-2; RANKL, receptor activator of NF- $\mathrm{B}$ ligand; OPG, osteoprotegerin; cytoD, cytochalasin D.

A

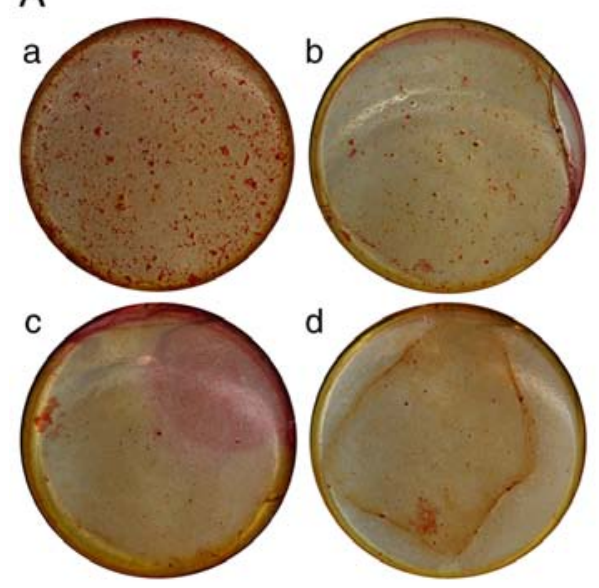

B
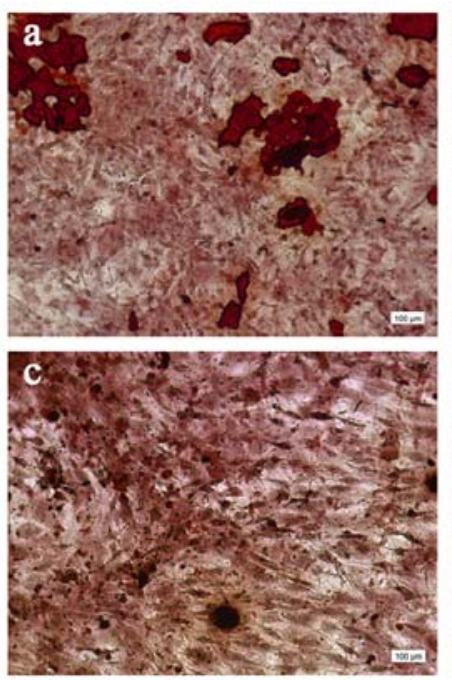
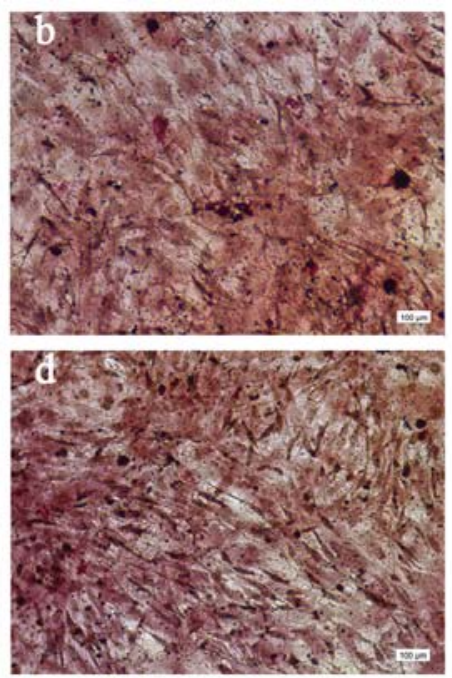

Figure 5. Culture supernatants collected from inhibitor-treated PDLCs exhibit reduced osteogenesis-promoting capacity after $8 \mathrm{~h}$ of loading. Osteoblasts that were cultured in the culture supernatant of PDLCs following loading exhibited differential capacities for mineralization. (A) Osteoblasts on plates: (a) Control group, (b) cytoD-pretreated group, (c) GsMTx4-treated group and (d) GSK205-treated group. (B) Osteoblasts under a light microscope: (a) Control group, (b) cytoD-pretreated group, (c) GsMTx4-treated group and (d) GSK205-treated group. Results suggested that the induction of osteoblast mineralization by loaded PDLCs could be suppressed by specific inhibitors. PDLC, periodontal ligament cell; cytoD, cytochalasin D.

terms of permeability, an ability to activate conditions and in their downstream signaling pathways.

That being considered, the MAPK family of signal transducing pathways consists of three major cascades, ERK, JNK and p38, all of which participate in cell activation by mechanical stress (45-47). However, it remains to be elucidated whether mechanical signals that are transduced by MSCs are indeed delivered by MAPK in PDLCs. It has been reported that the opening of Piezol induced by mechanical force can initiate chondrocyte apoptosis through ERK1 and ERK2 signaling pathways (20).

In the present study, no significant difference was found in the phosphorylation of ERK1/2 when the Piezol channel was inhibited. By contrast, a significant increase in glycogen synthase kinase (GSK) $3 \alpha / \beta$ phosphorylation was observed. As GSK3 is a negative regulator of ERK1/2 (48), this might imply that GsMTx4 downregulates the ERK1/2 signaling pathway via the activation of GSK. Additionally, GSK $3 \beta$ contributes to $\beta$-catenin activation via Ras suppression (49). Furthermore, GSK $3 \beta$ can also directly inhibit the transcription factors c-Fos and c-Jun (50). The results of microarray analysis also showed increased phosphorylation of JNK3, JNK Pan and MAPK kinase $(\mathrm{MKK}) 3$ and 6 after Piezol channel attenuation. Among these, MKK3 and 6 are members of the p38-mediated signaling pathway, which catalyze p38 phosphorylation (51). Further, the JNK signaling pathway can activate c-Jun (52). It 


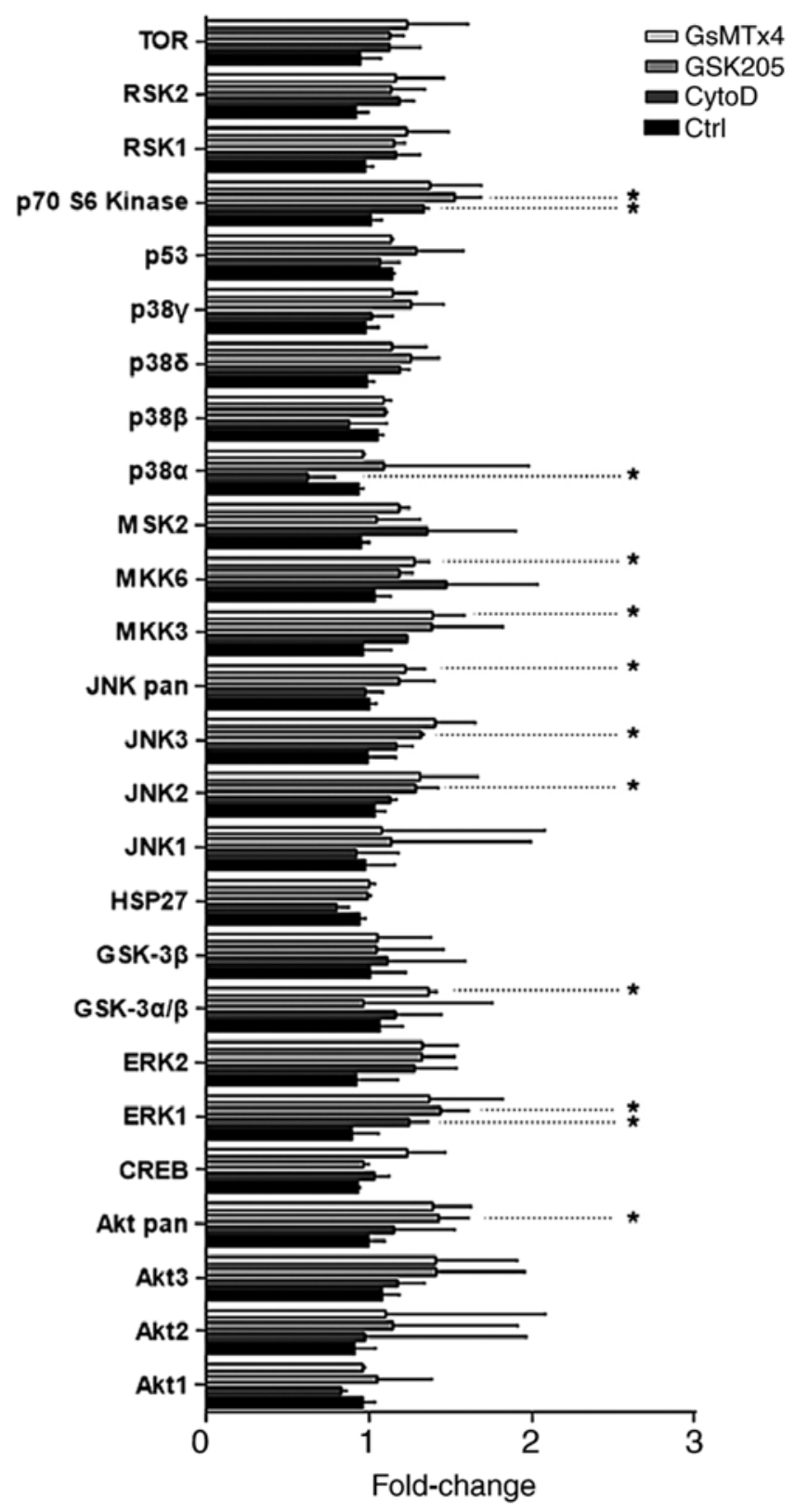

Figure 6. Fold change of phosphorylated-MAPK proteins in each group was compared with the control group. ${ }^{*} \mathrm{P}<0.05$ and variations of $>20 \%$ vs. Control. MAPK, mitogen-activated protein kinase; RSK, ribosomal S6 kinase; MSK, mitogen- and stress-activated kinase; MKK, MAPK kinase; HSP, heat shock protein; CREB, cAMP response element-binding protein.

might be hypothesized that when the Piezol channel is blocked, the ERK-related signaling pathway is also inhibited. Increased functional activation of both JNK and p38 may be attributable to cellular compensation mechanisms under conditions where the ERK signaling pathway was compromised.

In contrast to the Piezo1 channel, when the TRPV4 ion channel was blocked by GSK205, increased phosphorylation of ERK, JNK, AKT and p70S6 kinase was observed. In addition, initiation of the AKT pathway is known to result from mechanical stimuli (53). The absence of calcium in the extracellular matrix has been noted to attenuate AKT phosphorylation (54). Until now, evidence supporting an interaction between TRPV4 and the MAPK signaling pathway in mechanotransduction, especially in PDLCs, has been lacking.
It has been reported that blocking of the ERK1/2 signal transduction pathway may prevent increases in TRPV4 channel activity induced by hypoosmotic stress in chondrocytes (55). Conversely, the effects of TRPV4 inhibition on MAPK sub-members have rarely been reported. As, in the present study, no significant suppressive effect was observed in the context of MAPK signaling molecules, it might be concluded that the signals received and transduced by TRPV4 were not delivered via the MAPK-mediated signaling pathways.

Microarray analysis also revealed that phosphorylation of ERK and p70S6 was enhanced, whereas that of p38 $\alpha$ was diminished following loading and under conditions when the stress fibers were disrupted by cytoD. This observation suggests that p38 may participate in signal delivery via the cytoskeletal network. As reported by Qi et al (56), interference of stress fiber assembly by Rac1 knockdown may dampen p38 phosphorylation in vascular smooth muscle cells loaded by cyclic strain. Another study revealed the roles of p38 signaling in PDLC mechanotransduction loaded with mechanical stress (57).

There were certain limitations in the present study. Protein assays are an efficient way to screen out potential candidates in the mechanotransduction of PDLCs; the roles of the proteins need to be verified via western blotting or in vivo experiments. Furthermore, it has been established that several signaling molecules, including MAPK, Wnt and Notch, are involved in the process of mechanotransduction $(58,59)$; however, the present study only focused on the MAPK pathway. Due to insufficient time and financial constraints, verification experiments could not be performed in the present study. Further investigations into these pathways will be conducted in the future.

In summary, the present study revealed the roles of Piezol and TRPV4 in the mechanotransduction of hPDLCs. In addition, it was suggested that both Piezol and TRPV4 may impart their functional activities via different signaling pathways distinct from actin cytoskeletal rearrangement. Furthermore, the present study indicated that the ERK and p38 signaling pathways might participate in mechanotransduction mediated by the Piezol channel and actin cytoskeleton, respectively. However, a similar role was not observed for the TRPV4 channel. Other signal transduction pathways might be engaged as a compensatory mechanism under conditions when an individual pathway might be compromised.

\section{Acknowledgements}

The authors would like to thank Dr Weibing Zhang (Affiliated Stomatological Hospital of Nanjing Medical University, Nanjing, Jiangsu, China) for his assistance with the experiments.

\section{Funding}

This work was supported by the National Natural Science Foundation of China (grant nos. 81830031 and 81570959), the State Key Lab of Reproductive Medicine of Nanjing Medical University (grant no. JX116GSP20171416), the Priority Academic Program Development of Jiangsu Higher Education Institutions (grant no. PAPD-2018-87), the Natural Science Foundation of Jiangsu Province (grant nos. BL2014073 and 15KJA320002) and the Jiangsu Provincial Key Medical Discipline (grant no. zdxka2016026). 


\section{Availability of data and materials}

The datasets used and/or analyzed during the current study are available from the corresponding author on reasonable request.

\section{Authors' contributions}

YS and LW were responsible for the study design and implementation. SG and LS performed the cell culture, mechanical loading, patient consent and documentation. PCR, western blotting and data analysis were conducted by $\mathrm{CZ}$ and YP. YS was a major contributor in writing the manuscript. All authors read and approved the final manuscript.

\section{Ethics approval and consent to participate}

Informed written consent was obtained from the legal guardians of all donors. All experimental protocols were approved by the Ethics Committee of Shanghai Tenth People's Hospital [policy nos. 2008 (20) and SHDSYY-2017-2473].

\section{Patient consent for publication}

Not applicable.

\section{Competing interests}

The authors declare that they have no competing interests.

\section{References}

1. Proffit WR, Fields HW and Sarver DM (eds): Contemporary Orthodontics. 5th edition. Mosby, St. Louis, 2012.

2. Chang M, Lin $\mathrm{H}, \mathrm{Fu} H$, Wang $B$, Han $G$ and Fan $M$ : MicroRNA-195-5p regulates osteogenic differentiation of periodontal ligament cells under mechanical loading. J Cell Physiol 232: 3762-3774, 2017.

3. Feng L, Zhang Y, Kou X, Yang R, Liu D, Wang X, Song Y, Cao H, He D, Gan Y and Zhou Y: Cadherin-11 modulates cell morphology and collagen synthesis in periodontal ligament cells under mechanical stress. Angle Orthod 87: 193-199, 2017.

4. Zhang $\mathrm{H}$ and Zhang D: Effects of periodontal ligament cells on alveolar bone metabolism under the action of force and inflammatory factors and its molecular mechanisms. Zhongguo Yi Xue Ke Xue Yuan Xue Bao 39: 432-437, 2017.

5. Li Y, Jacox LA, Little SH and Ko CC: Orthodontic tooth movement: The biology and clinical implications. Kaohsiung J Med Sci 34: 207-214, 2018.

6. Roca-Cusachs P, del Rio A, Puklin-Faucher E, Gauthier NC, Biais N and Sheetz MP: Integrin-dependent force transmission to the extracellular matrix by alpha-actinin triggers adhesion maturation. Proc Natl Acad Sci USA 110: E1361-E1370, 2013.

7. Zhou J, Aponte-Santamaria C, Sturm S, Bullerjahn JT, Bronowska A and Grater F: Mechanism of focal adhesion kinase mechanosensing. PLoS Comput Biol 11: e1004593, 2015.

8. Jin Y, Li J, Wang Y, Ye R, Feng X, Jing Z and Zhao Z: Functional role of mechanosensitive ion channel Piezol in human periodontal ligament cells. Angle Orthod 85: 87-94, 2015.

9. Lu J, Lee YK, Ran X, Lai WH, Li RA, Keung W, Tse K, Tse HF and Yao X: An abnormal TRPV4-related cytosolic $\mathrm{Ca}^{2+}$ rise in response to uniaxial stretch in induced pluripotent stem cells-derived cardiomyocytes from dilated cardiomyopathy patients. Biochim Biophys Acta Mol Basis Dis 1863: 2964-2972, 2017.

10. Cox CD, Bae C, Ziegler L, Hartley S, Nikolova-Krstevski V, Rohde PR, Ng CA, Sachs F, Gottlieb PA and Martinac B: Removal of the mechanoprotective influence of the cytoskeleton reveals PIEZO1 is gated by bilayer tension. Nat Commun 7: 10366, 2016.
11. Coste B, Mathur J, Schmidt M, Earley TJ, Ranade S, Petrus MJ, Dubin AE and Patapoutian A: Piezo1 and Piezo2 are essential components of distinct mechanically activated cation channels. Science 330: 55-60, 2010

12. Ge J, Li W, Zhao Q, Li N, Chen M, Zhi P, Li R, Gao N, Xiao B and Yang M: Architecture of the mammalian mechanosensitive Piezol channel. Nature 527: 64-69, 2015.

13. Strotmann R, Harteneck C, Nunnenmacher K, Schultz G and Plant TD: OTRPC4, a nonselective cation channel that confers sensitivity to extracellular osmolarity. Nat Cell Biol 2: 695-702, 2000.

14. Güler AD, Lee H, Iida T, Shimizu I, Tominaga M and Caterina M: Heat-evoked activation of the ion channel, TRPV4. J Neurosci 22: 6408-6414, 2002.

15. Gao X, Wu L and O'Neil RG: Temperature-modulated diversity of TRPV4 channel gating: Activation by physical stresses and phorbol ester derivatives through protein kinase C-dependent and -independent pathways. J Biol Chem 278: 27129-27137, 2003.

16. Danielczok JG, Terriac E, Hertz L, Petkova-Kirova P, Lautenschlager F, Laschke MW and Kaestner L: Red blood cell passage of small capillaries is associated with transient $\mathrm{Ca}^{2+}$-mediated adaptations. Front Physiol 8: 979, 2017.

17. Servin-Vences MR, Richardson J, Lewin GR and Poole K: Mechanoelectrical transduction in chondrocytes. Clin Exp Pharmacol Physiol 45: 481-488, 2018.

18. Sugimoto A, Miyazaki A, Kawarabayashi K, Shono M, Akazawa Y, Hasegawa T, Ueda-Yamaguchi K, Kitamura T, Yoshizaki K, Fukumoto S and Iwamoto T: Piezo type mechanosensitive ion channel component 1 functions as a regulator of the cell fate determination of mesenchymal stem cells. Sci Rep 7: 17696, 2017.

19. Blythe NM, Muraki K, Ludlow MJ, Stylianidis V, Gilbert HTJ, Evans EL, Cuthbertson K, Foster R, Swift J, Li J, et al: Mechanically activated Piezol channels of cardiac fibroblasts stimulate p38 mitogen-activated protein kinase activity and interleukin-6 secretion. J Biol Chem 294: 17395-17408, 2019.

20. Li XF, Zhang Z, Li XD, Wang TB and Zhang HN: Mechanism of the Piezol protein-induced apoptosis of the chondrocytes through the MAPK/ERK1/2 signal pathway. Zhonghua Yi Xue Za Zhi 96: 2472-2477, 2016 (In Chinese).

21. Liang J, Huang B, Yuan G, Chen Y, Liang F, Zeng H, Zheng S, Cao L, Geng D and Zhou S: Stretch-activated channel Piezol is up-regulated in failure heart and cardiomyocyte stimulated by AngII. Am J Transl Res 9: 2945-2955, 2017.

22. Qu YJ, Zhang X, Fan ZZ, Huai J, Teng YB, Zhang Y and Yue SW: Effect of TRPV4-p38 MAPK pathway on neuropathic pain in rats with chronic compression of the dorsal root ganglion. Biomed Res Int 2016: 6978923, 2016.

23. Livak KJ and Schmittgen TD: Analysis of relative gene expression data using real-time quantitative PCR and the 2(-Delta Delta C(T)) method. Methods 25: 402-408, 2001.

24. Yang Z, Tan S, Shen Y, Chen R, Wu C, Xu Y, Song Z and Fu Q: Inhibition of FSS-induced actin cytoskeleton reorganization by silencing LIMK2 gene increases the mechanosensitivity of primary osteoblasts. Bone 74: 182-190, 2015.

25. Kanzaki H, Chiba M, Shimizu Y and Mitani H: Periodontal ligament cells under mechanical stress induce osteoclastogenesis by receptor activator of nuclear factor kappaB ligand up-regulation via prostaglandin E2 synthesis. J Bone Miner Res 17: 210-220, 2002.

26. Boyce BF and Xing L: The RANKL/RANK/OPG pathway. Curr Osteoporos Rep 5: 98-104, 2007.

27. Garlet TP, Coelho U, Silva JS and Garlet GP: Cytokine expression pattern in compression and tension sides of the periodontal ligament during orthodontic tooth movement in humans. Eur J Oral Sci 115: 355-362, 2007.

28. Kim T, Handa A, Iida J and Yoshida S: RANKL expression in rat periodontal ligament subjected to a continuous orthodontic force. Arch Oral Biol 52: 244-250, 2007.

29. Kanjanamekanant K, Luckprom P and Pavasant P: Mechanical stress-induced interleukin-1beta expression through adenosine triphosphate/P2X7 receptor activation in human periodontal ligament cells. J Periodontal Res 48: 169-176, 2013.

30. Fu HD, Wang BK, Wan ZQ, Lin H, Chang ML and Han GL: Wnt5a mediated canonical Wnt signaling pathway activation in orthodontic tooth movement: Possible role in the tension force-induced bone formation. J Mol Histol 47: 455-466, 2016.

31. Alhashimi N, Frithiof L, Brudvik P and Bakhiet M: CD40-CD40L expression during orthodontic tooth movement in rats. Angle Orthod 74: 100-105, 2004. 
32. Grieve WG 3rd, Johnson GK, Moore RN, Reinhardt RA and DuBois LM: Prostaglandin E (PGE) and interleukin-1 beta (IL-1 beta) levels in gingival crevicular fluid during human orthodontic tooth movement. Am J Orthod Dentofacial Orthop 105: 369-374, 1994.

33. Dougall WC: Molecular pathways: Osteoclast-dependent and osteoclast-independent roles of the RANKL/RANK/OPG pathway in tumorigenesis and metastasis. Clin Cancer Res 18: 326-335, 2012

34. Sachs F: Mechanical transduction by ion channels: A cautionary tale. World J Neurol 5: 74-87, 2015.

35. Ingber DE: Tensegrity-based mechanosensing from macro to micro. Prog Biophys Mol Biol 97: 163-179, 2008.

36. Li J, Chen G, Zheng L, Luo S and Zhao Z: Osteoblast cytoskeletal modulation in response to compressive stress at physiological levels. Mol Cell Biochem 304: 45-52, 2007.

37. Mammoto A and Ingber DE: Cytoskeletal control of growth and cell fate switching. Curr Opin Cell Biol 21: 864-870, 2009.

38. Son GY, Yang YM, Park WS, Chang I and Shin DM: Hypotonic stress induces RANKL via transient receptor potential melastatin 3 (TRPM3) and vaniloid 4 (TRPV4) in human PDL cells. J Dent Res 94: 473-481, 2015.

39. Choquet D, Felsenfeld DP and Sheetz MP: Extracellular matrix rigidity causes strengthening of integrin-cytoskeleton linkages. Cell 88: 39-48, 1997.

40. Matthews BD, Thodeti CK, Tytell JD, Mammoto A, Overby DR and Ingber DE: Ultra-rapid activation of TRPV4 ion channels by mechanical forces applied to cell surface betal integrins. Integr Biol (Camb) 2: 435-442, 2010.

41. Berridge MJ, Bootman MD and Lipp P: Calcium-a life and death signal. Nature 395: 645-648, 1998.

42. Berridge MJ,Lipp P and Bootman MD: The versatility and universality of calcium signalling. Nat Rev Mol Cell Biol 1: 11-21, 2000.

43. Benavides Damm T and Egli M: Calcium's role in mechanotransduction during muscle development. Cell Physiol Biochem 33: 249-272, 2014.

44. Strotmann R, Schultz G and Plant TD: Ca2+-dependent potentiation of the nonselective cation channel TRPV4 is mediated by a C-terminal calmodulin binding site. J Biol Chem 278: 26541-26549, 2003

45. Ishida T, Peterson TE, Kovach NL and Berk BC: MAP kinase activation by flow in endothelial cells. Role of beta 1 integrins and tyrosine kinases. Circ Res 79: 310-316, 1996.

46. Kippenberger S, Bernd A, Loitsch S, Guschel M, Muller J, Bereiter-Hahn J and Kaufmann R: Signaling of mechanical stretch in human keratinocytes via MAP kinases. J Invest Dermatol 114 408-412, 2000

47. Yamazaki T, Komuro I, Shiojima I and Yazaki Y: The molecular mechanism of cardiac hypertrophy and failure. Ann N Y Acad Sci 874: 38-48, 1999.

48. Wang Q, Zhou Y, Wang X and Evers BM: Glycogen synthase kinase-3 is a negative regulator of extracellular signal-regulated kinase. Oncogene 25: 43-50, 2006.
49. Liu S, Fang X, Hall H, Yu S, Smith D, Lu Z, Fang D, Liu J, Stephens LC, Woodgett JR and Mills GB: Homozygous deletion of glycogen synthase kinase 3beta bypasses senescence allowing Ras transformation of primary murine fibroblasts. Proc Nat Acad Sci USA 105: 5248-5253, 2008.

50. Götschel F, Kern C, Lang S, Sparna T, Markmann C, Schwager J, McNelly S, von Weizsäcker F, Laufer S, Hecht A and Merfort I: Inhibition of GSK3 differentially modulates NF-kappaB, CREB, AP-1 and beta-catenin signaling in hepatocytes, but fails to promote TNF-alpha-induced apoptosis. Exp Cell Res 314: 1351-1366, 2008.

51. Terada Y, Nakashima O, Inoshita S, Kuwahara M, Sasaki S and Marumo F: Mitogen-activated protein kinase cascade and transcription factors: The opposite role of MKK3/6-p38K and MKK1-MAPK. Nephrol Dial Transplant 14 (Suppl 1): S45-S47, 1999.

52. Papadopoulou A, Todaro A, Eliades T and Kletsas D: Effect of hyperglycaemic conditions on the response of human periodontal ligament fibroblasts to mechanical stretching. Eur J Orthod 41: 583-590, 2019.

53. Danciu TE, Adam RM, Naruse K, Freeman MR and Hauschka PV: Calcium regulates the PI3K-Akt pathway in stretched osteoblasts. FEBS Lett 536: 193-197, 2003.

54. Divolis G, Mavroeidi P, Mavrofrydi O and Papazafiri P. Differential effects of calcium on PI3K-Akt and HIF-1 $\alpha$ survival pathways. Cell Biol Toxicol 32: 437-449, 2016.

55. Hdud IM, Mobasheri A and Loughna PT: Effect of osmotic stress on the expression of TRPV4 and BKCa channels and possible interaction with ERK1/2 and p38 in cultured equine chondrocytes. Am J Physiol Cell Physiol 306: C1050-C1057, 2014

56. Qi YX, Qu MJ, Yan ZQ, Zhao D, Jiang XH, Shen BR and Jiang ZL: Cyclic strain modulates migration and proliferation of vascular smooth muscle cells via Rho-GDIalpha, Rac1, and p38 pathway. J Cell Biochem 109: 906-914, 2010.

57. Zheng L, Huang Y, Song W, Gong X, Liu M, Jia X, Zhou G, Chen L, Li A and Fan Y: Fluid shear stress regulates metalloproteinase-1 and 2 in human periodontal ligament cells: Involvement of extracellular signal-regulated kinase (ERK) and P38 signaling pathways. J Biomech 45: 2368-2375, 2012.

58. Chen Y, Yang K, Zhou Z, Wang L, Du Y and Wang X: Mechanical stress modulates the RANKL/OPG system of periodontal ligament stem cells via $\alpha 7 \mathrm{nAChR}$ in human deciduous teeth: An in vitro study. Stem Cells Int 2019: 5326341, 2019.

59. Manokawinchoke J, Pavasant P and Osathanon T: Intermittent compressive stress regulates Notch target gene expression via transforming growth factor- $\beta$ signaling in murine pre-osteoblast cell line. Arch Oral Biol 82: 47-54, 2017.

(i) (2) This work is licensed under a Creative Commons Attribution-NonCommercial-NoDerivatives 4.0 International (CC BY-NC-ND 4.0) License. 